\title{
Effect of Nurse-led Program in Coronary Heart Diseases Patients: A Systematic Review
}

\author{
Al Afik ${ }^{1,2 *}$, Nursalam Nursalam ${ }^{3}$, A. Yuni Sufyanti ${ }^{3}$, Riza Fikriana ${ }^{4}$ \\ ${ }^{1}$ Student of Nursing Doctoral Program, Faculty of Nursing, Universitas Airlangga, Surabaya, Indonesia; ${ }^{2}$ Nursing Program, \\ Faculty of Medicine and Health Science, Universitas Muhammadiyah Yogayakarta, Bantul, Indonesia; ${ }^{3}$ Department of Nursing \\ Management, Faculty of Nursing, University of Airlangga, Surabaya, Indonesia; ${ }^{4}$ Department of Emergency, Sekolah Tinggi \\ IImu Kesehatan Kepanjen, Malang, Indonesia
}

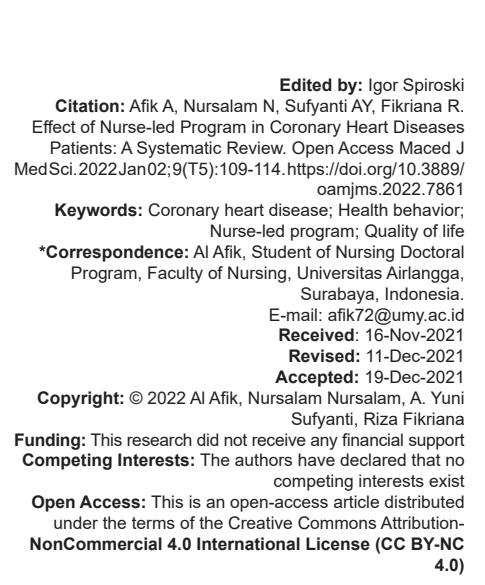

\section{Abstract}

BACKGROUND: Coronary Heart Diseases (CHD) is one of the leading causes of death worldwide. Proper management for $\mathrm{CHD}$ patients is needed to reduce mortality. Nurses have a very important role in making $\mathrm{CHD}$ patients can carry out optimal health behaviors.

AIM: This review study aims to identify the effect of the nurse-led program on CHD patients.

METHODS: This systematic review was based on four electronic databases (Scopus, Science Direct, ProQuest, SAGE Journals) and published between 2011 and 2020. This review used the Joanna Briggs Institute and Prisma guidelines. The study's eligibility was assessed from the title, abstract, research methodology, and full text. The review results were presented in tabulated data and narrative form.

RESULTS: Nine articles showed that the nurse-led program significantly improved health behavior, health-related quality of life, clinical outcome, and illness perception in CHD patients.

CONCLUSIONS: It is expected that the improvement of management in CHD patients will improve patients' quality of life. Hence, it could reduce the number of morbidities and mortality.

\section{Introduction}

Acute Coronary Syndrome (ACS) is part of coronary heart disease (a cardiovascular problem), one of the causes of mortality worldwide. The recent coronary heart disease mortality rate increased from 2006 to 2016 by $19.0 \%$, namely 7.96 million to 9.48 million [1]. Furthermore, management of medication and proper care is needed for someone suffering from coronary heart disease to minimize the risk of death. The management of patients with coronary heart disease has two main

\section{Objectives}

Reducing symptoms and preventing recurrence and mortality [2]. Clinical management can be carried out by providing medication, lifestyle modification, and weight management [3].

Self-care behavior in patients with coronary heart disease can help reduce the appearance of signs and symptoms of the disease, which can improve the patient's health status. Otherwise, inadequate self-care will result in poor patient health, increasing patient recurrence [4], [5]. Meanwhile, adequate self-care can increase satisfaction, fulfill daily needs, manage stress, and reduce complications [6].

Nurses are health service providers who have a very important role in managing patients with coronary heart disease. Nursing interventions can promote cardiovascular health by properly conducting counseling and self-management [7]. Patients with ACS are expected to have self-management skills in preventing recurrences that can lead to emergencies. However, the involvement of nurses is still not optimal in helping patients with ACS.

Nurse-led programs are a form of nursing intervention given to patients with coronary heart disease or ACS. Nurse-led programs can improve patient health outcomes [8]. This review, subsequently, aims to identify the effect of a non-led program in patients with coronary heart disease. 


\section{Methods}

\section{Study protocol}

The study protocol used the Joanna Briggs Institute Guideline as a guide for assessing the quality of the study and used the PRISMA checklist to determine which articles fit for the purpose.

\section{Searching strategy}

The selected articles in this systematic review were obtained by searching electronic databases, including Scopus, Science Direct, ProQuest, SAGE Journals. The keywords used to select eligible articles in this review that followed Medical Subject Heading included ("Coronary Heart Diseases (CHD)" OR "Coronary Artery Diseases" OR "ACS" OR "Acute Myocardial Infarction") AND ("Health Behavior" OR "Lifestyle") AND ("Health Status) AND ("Nurse-led Program).

\section{Inclusion criteria}

The stages in selecting articles started by reading the appropriate article. Furthermore, the abstract was read mainly on our research design, the sample selected, and the analyzed data. After that, the researchers opened the full text of the article by checking the suitability of the predetermined inclusion criteria. The inclusion criteria used Population, Intervention, Comparability, Outcome, Study Design framework. The population included patients who have been diagnosed with coronary heart disease/coronary artery diseases/ACS/acute myocardial infarction, intervention Community base or prehospital received treatment in the form of nurse-led program. There were a comparison group (control)and an intervention outcome. Other eligibility criteria were full-text access, published in 2011-2020 and English. The Joanna Briggs Institute critical appraisal tool was also used as a checklist for Randomized Controlled Trials review.

The results of the selection of articles obtained 9 articles that met the inclusion criteria. The article selection was illustrated in the flow diagram based on PRISMA 2009 below, as showed in Figure 1.

\section{Results}

\section{Characteristic of study}

The results of the review obtained a study of nine articles that met the inclusion criteria. All studies used a randomized controlled trial design. Participants were the

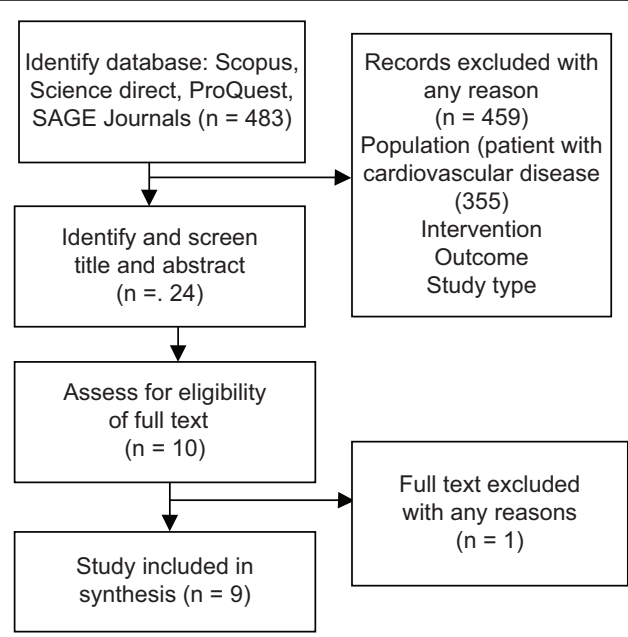

Figure 1: The flow diagram based on Prisma bellow

patients who had been diagnosed with $\mathrm{CHD}(\mathrm{n}=1)$, coronary artery diseases $(n=4)$, ACS $(n=4)$, and acute myocardial infarction $(n=1)$. All participants in the treatment group received intervention in the form of a nurse-led program, in which eight studies were conducted independently by nurses, and one study was conducted in collaboration with other health workers. Meanwhile, the entire control group received follow-ups or usual care routines.

\section{Outcomes of study}

As a result of the intervention, the study's outcome found that the nurse-led program had a significant effect in improving health behavior, health-related quality of life ( $\mathrm{HrQoL})$, cognitive and psychological factors, and clinical outcomes.

The literature search results are listed in Table 1.

1. Nurse-led programs improve health behavior.

Fivestudies $(1,3,4,5,6)$ showed thatnurse-led program intervention was able to improve health behavior in patients. Self-management, which is part of nurselead to Improved health behavior, could be in the form of exercise frequency and time, healthy diet, stress management, smoking cessation, adherence management, emergency management, emergency visits, outpatient visits, medication adherence, and alcohol consumption.

2. Nurse-led programs improve HrQoL.

Four studies $(2,3,4,8)$ described the effectiveness of nurse-led programs in increasing HrQoL in patients with CHD. The increase in HrQoL is in the form of increased physical health status (physical function and bodily pain) and mental (emotional role function, mental health, vitality, and role limitationsemotional). A reduction in depressive symptoms and an improvement in the social subscale were also observed in one study.

3. Nurse-led programs improve cognitive and psychological factors. 


\section{Table 1: The result of systematic review}

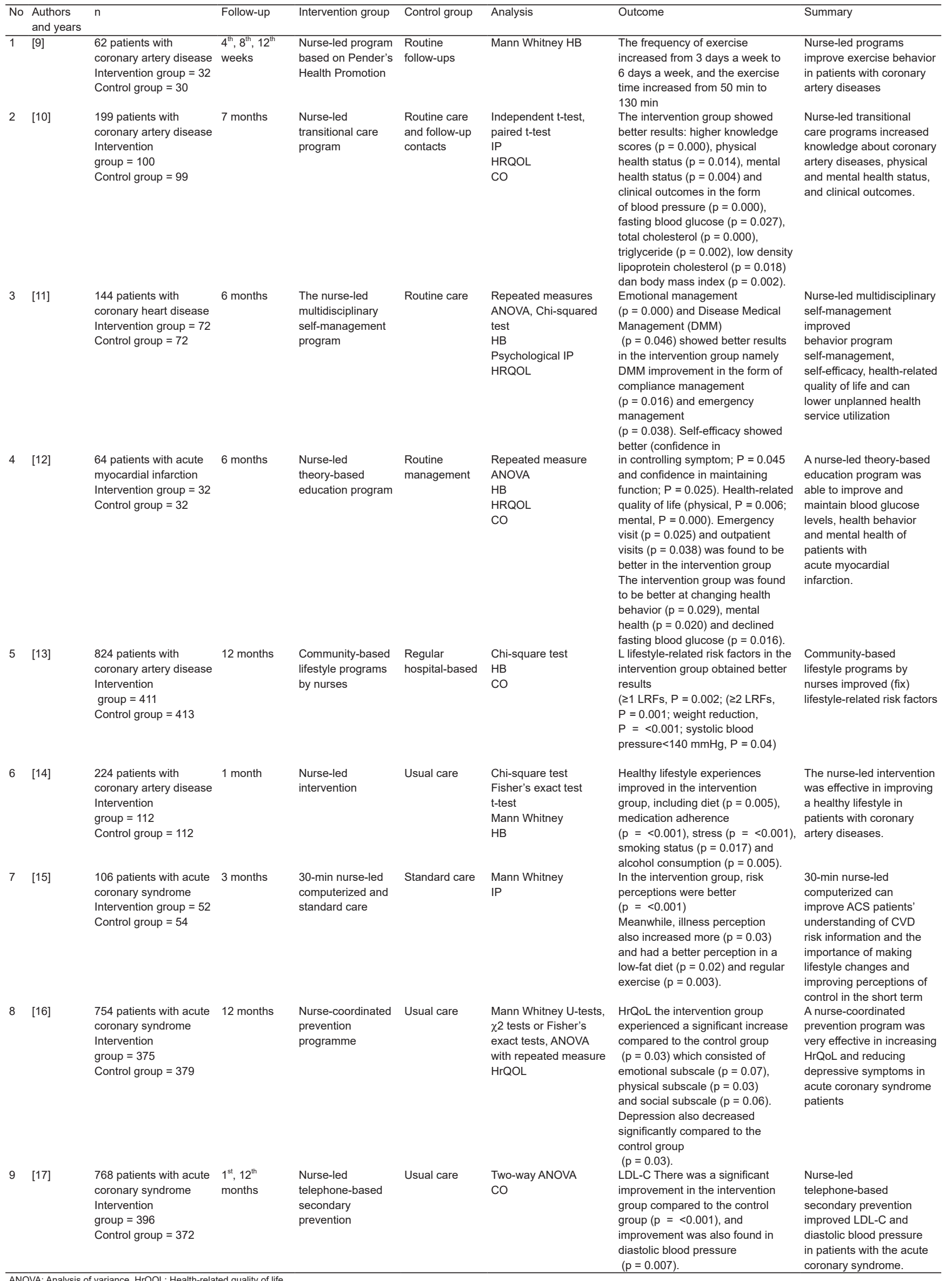


Nurse-led programs effectively increase knowledge and self-efficacy in controlling symptoms and maintaining function, risk, and illness perception.

4. Nurse-led programs improve Clinical Outcomes.

Clinical outcomes are one of the effects obtained from nurse-led program interventions in four studies $(2,4,5,9)$. Nurse-led programs improve blood pressure, fasting blood glucose, total cholesterol, triglycerides, low-density lipoprotein cholesterol, body mass index, and weight reduction.

\section{Discussion}

Coronary heart disease is a disease that needs to be followed up immediately. Proper management is needed to minimize complications. As one of the health service providers, the nurse must provide professional nursing care to optimize the patient's health status. A nurse-led program is a service program that nurses can carry out to maximize the nursing care provided. This program is an intervention carried out by nurses to manage the patient's illness [18]. Some of the advantages of providing this program are reducing the burden of treatment, improving integrated chronic disease management [19], [20] and improving self-care skills [21]. Disease recurrence can also be reduced through this program [19].

In this systematic review, our purpose was to identify the effect of a nurse-led program intervention in patients with coronary heart disease. The results of nine studies found that the nurse-led program effectively improved health behavior, HrQoL, cognitive and psychological factors, and clinical outcomes. Healthy living behavior and adherence to self-care could be improved by conducting nurse-led program intervention. The results of this review are in line with other studies in which the nurse-led program can improve health behavior in the form of medication adherence to patients suffering from chronic heart failure [22] and health-promoting behavior in patients with metabolic syndrome [23]. Diet regulating behavior and increased exercise were also found to be better [24]. However, it contradicts the results of other studies where the nurse-led program did not result in lifestyle improvements since everyone has a different lifestyle and has inaccurate beliefs [25].

HrQoL, both physical and mental health status, has increased due to nurse-led programs. The results of the review are in line with other studies where the physical aspects had significantly improved, resulting in a decrease in patients undergoing hospitalization or visits to emergency services [20], the occurrence of psychosocial improvement [24], and improving depression symptoms in older adults and early-stage cancer patients [26], [27]. Skill practice obtained in nurse-led intervention can help manage anxiety and fear [28]. However, other studies showed a different matter, where the nurse-led program did not cause a decrease in anxiety and depression in people with coronary heart disease so that it did not improve the mental health status of the sufferer [29]. The absence of improvement in mental health is also supported by other studies conducted on post-percutaneous coronary intervention patients [30].

Cognitive and psychological factors in the form of increased knowledge, self-efficacy in controlling symptoms and maintaining the function, and risk and illness perception experienced improvement due to nurse-led intervention. This review is in line with other studies that showed the effectiveness of nurse-led programs in increasing self-efficacy [23], improving risk perception [25], and effectively increasing knowledge and early detection beliefs in cancer patients [31].

Changes in clinical outcomes due to the provision of nurse-led programs are shown from the results of this review. These results align with other studies that a decrease in blood pressure and cholesterol occurred due to nurse-led program interventions; thus, it reduced the risk of cardiovascular disease [32], [33] and improved healthy habits [34]. These improvements in clinical outcomes may reduce patient recurrence to reduce visits to emergency services and hospitalizations.

\section{Conclusion}

Based on this study's result, it can be concluded that nurse-led programs were part of nursing interventions that were very effective for patients with coronary heart disease. The effectiveness of this intervention was based on the results of a review that the nurse-led program could improve health behavior, HrQoL, cognitive and psychological factors and improve clinical outcomes in patients. The practical implication of this result is that nurses can use this program to maximize and optimize nursing care for patients with coronary heart disease.

\section{References}

1. Naghavi M, Abajobir A, Cristiana A, Abbas KM, Abd-Allah F, Abera SF, et al. Global, regional, and national age-sex specific mortality for 264 causes of death, 1980-2016: A systematic analysis for the Global Burden of Disease Study 2016. Lancet. 2017;390(10100):1151-210. https://doi.org/10.1016/ S0140-6736(17)32152-9 
2. Cassar A, Holmes DR, Rihal CS, Gersh BJ. Chronic coronary artery disease: Diagnosis and management. Mayo Clin Proc. 2009;84(12):1130-46. https://doi.org/10.4065/mcp.2009.0391 PMid: 19955250

3. Arnold SV, Bhatt DL, Barsness GW, Beatty AL, Deedwania PC, Inzucchi SE, et al. Clinical management of stable coronary artery disease in patients with Type 2 diabetes mellitus: A scientific statement from the American Heart Association. Circulation. 2020;141(19):E779-806. https://doi.org/10.1161/ CIR.0000000000000766 PMid:32279539

4. Niakan M, Paryad E, Leili EK, Sheikholeslami F. Depressive symptoms effect on self care behavior during the first month after myocardial infarction. Glob J Health Sci. 2015;7(4):382-91. https://doi.org/10.5539/gjhs.v7n4p382

\section{PMid:25946944}

5. Adib-Hajbaghery M, Maghaminejad F, Abbasi A. The role of continuous care in reducing readmission for patients with heart failure. J Caring Sci. 2013;2(4):255-67. https://doi.org/10.5681/ jcs.2013.031

\section{PMid:25276734}

6. Lycholip E, Aamodt IT, Lie I, Šimbelytė T, Puronaitė R, Hillege H et al. The dynamics of self-care in the course of heart failure management: Data from the IN TOUCH study. Patient Prefer Adherence. 2018;12:1113-22. https://doi.org/10.2147/PPA. S162219

\section{PMid:29983549}

7. Hayman LL, Berra K, Fletcher BJ, Miller NH. The role of nurses in promoting cardiovascular health worldwide: The global cardiovascular nursing leadership forum. J Am Coll Cardiol. 2015;66(7):864-6. https://doi.org/10.1016/j.jacc.2015.06.1319 PMid:26271070

8. Son YJ, Choi J, Lee HJ. Effectiveness of nurse-led heart failure self-care education on health outcomes of heart failure patients: A systematic review and meta-analysis. Int J Environ Res Public Health. 2020;17(18):6559. https://doi.org/10.3390/ ijerph17186559

PMid:32916907

9. Karataş T, Polat U. Effect of nurse-led program on the exercise behavior of coronary artery patients: Pender's health promotion model. Patient Educ Couns. 2021;104(5):1183-92. https://doi. org/10.1016/j.pec.2020.10.003

PMid:33129630

10. Zhang P, Hu YD, Xing FM, Li CZ, Lan WF, Zhang XL. Effects of a nurse-led transitional care program on clinical outcomes, healthrelated knowledge, physical and mental health status among Chinese patients with coronary artery disease: A randomized controlled trial. Int J Nurs Stud. 2016;74:34-43. https://doi. org/10.1016/j.jinurstu.2017.04.004

PMid:28601691

11. Jiang W, Zhang Y, Yan F, Liu H, Gao R. Effectiveness of a nurseled multidisciplinary self-management program for patients with coronary heart disease in communities: A randomized controlled trial. Patient Educ Couns. 2020;103(4):854-63. https://doi. org/10.1016/j.pec.2019.11.001

PMid:31727391

12. Park M, Song R, Jeong JO. Effect of goal attainment theory based education program on cardiovascular risks, behavioral modification, and quality of life among patients with first episode of acute myocardial infarction: Randomized study. Int J Nurs Stud. 2017;71:8-16. https://doi.org/10.1016/j. ijnurstu.2017.02.019 PMid:28279854

13. Minneboo M, Lachman S, Snaterse M, Jørstad HT, Riet GT, Boekholdt SM, et al. Community-based lifestyle intervention in patients with coronary artery disease: The RESPONSE-2 trial. J Am Coll Cardiol. 2017;70(3):318-27. https://doi.org/10.1016/j. jacc.2017.05.041

PMid:28705312

14. Gaudel P, Neupane S, Koivisto AM, Kaunonen M, Rantanen A Effects of a lifestyle-related risk factor modification intervention on lifestyle changes among patients with coronary artery disease in Nepal. Patient Educ Couns. 2020;104(6):1406-14. https://doi.org/10.1016/j.pec.2020.11.030

15. Broadbent E, Leggat A, Mclachlan A, Kerr A. Providing cardiovascular risk management information to acute coronary syndrome patients: A randomized trial. $\mathrm{Br} J$ Health Psychol. 2012;18(1):83-96. https://doi. org/10.1111/j.2044-8287.2012.02081.x PMid:22709363

16. Jørstad HT, Minneboo M, Helmes HJ, Fagel ND, Reimer WJ, Tijssen JG, et al. Effects of a nurse-coordinated prevention programme on health-related quality of life and depression in patients with an acute coronary syndrome: Results from the RESPONSE randomised controlled trial. BMC Cardiovasc Disord. 2016;16(1):144. https://doi.org/10.1186/ s12872-016-0321-4

PMid:27391321

17. Huber D, Henriksson R, Jakobsson S, Mooe T. Nurse-led telephone-based follow-up of secondary prevention after acute coronary syndrome: One-year results from the randomized controlled NAILED-ACS trial. PLoS One. 2017;12(9):e0183963. https://doi.org/10.1371/journal.pone.0183963

PMid:28886083

18. Chowdhury S, Stephen C, Mclnnes S, Halcomb E. Nurseled interventions to manage hypertension in general practice: A systematic review protocol. Collegian. 2020;27(3):340-3. https://doi.org/10.1016/j.colegn.2019.10.004

19. Rice H, Say R, Betihavas V. The effect of nurse-led education on hospitalisation, readmission, quality of life and cost in adults with heart failure. A systematic review. Patient Educ Couns. 2018;101(3):363-74. https://doi.org/10.1016/j.pec.2017.10.002 PMid:29102442

20. Bonner A, Havas K, Stone C, Abel J, Barnes M, Tam V, Douglas C. A multimorbidity nurse practitioner-led clinic: Evaluation of health outcomes. Collegian. 2020;27(4):430-6. https://doi.org/10.1016/j.colegn.2019.11.010

21. Chew HS, Sim KL, Hoi KC, Chair SY. Effectiveness of a nurse-led temporal self-regulation theory-based program on heart failure self-care: A randomized controlled trial. Int J Nurs Stud. 2021;2021:103872. https://doi.org/10.1016/j. ijnurstu.2021.103872

PMid:33516047

22. You J, Wang S, Li J, Luo Y. Usefulness of a nurse-led program of care for management of patients with chronic heart failure. Med Sci Monit. 2020;26:e920469. https://doi.org/10.12659/ MSM.920469 PMid:32068197

23. Zheng X, Yu H, Qu X, Chair SY, Wong EM, Wang Q. The effects of a nurse-led lifestyle intervention program on cardiovascular risk, self-efficacy and health promoting behaviours among patients with metabolic syndrome: Randomized controlled trial. Int J Nurs Stud. 2020;109:103638. https://doi.org/10.1016/j. ijnurstu.2020.103638

24. Williams B, Corless L, Abouda G. FRI-420-A nurse-led advice and lifestyle intervention shows high levels of patient-reported satisfaction and motivation in community-based management of NAFLD. J Hepatol. 2019;70(1):e579. https://doi.org/10.1016/ S0618-8278(19)31158-2

25. Koelewijn-van Loon MS, van der Weijden T, Ronda G, van Steenkiste B, Winkens B, Elwyn G, Grol R. Improving lifestyle 
and risk perception through patient involvement in nurseled cardiovascular risk management: A cluster-randomized controlled trial in primary care. Prev Med (Baltim). 2010;50(1):3544. https://doi.org/10.1016/j.ypmed.2009.11.007

PMid: 19944713

26. Wong AK, Wong FK. The psychological impact of a nurseled proactive self-care program on independent, non-frail community-dwelling older adults: A randomized controlled trial. Int J Nurs Stud. 2020;110:103724. https://doi.org/10.1016/j. ijnurstu.2020.103724

PMid:32777605

27. Shi Y, Cai J, Wu Z, Jiang L, Xiong G, Gan X, et al. Effects of a nurse-led positive psychology intervention on sexual function, depression and subjective well-being in postoperative patients with early-stage cervical cancer: A randomized controlled trial. Int J Nurs Stud. 2020;111:103768. https://doi.org/10.1016/j. ijnurstu.2020.103768

PMid:32971449

28. Reb AM, Borneman T, Economou D, Cangin MA, Cope DG, $\mathrm{Ma} \mathrm{H}$, Ruel N, et al. A nurse-led intervention for fear of cancer progression in advanced cancer: A pilot feasibility study. Eur J Oncol Nurs. 2020;49:101855. https://doi.org/10.1016/j. ejon.2020.101855

PMid:33120211

29. Luo ZC, Zhai L, Dai X. Does a nurse-led program of support and lifestyle management for patients with coronary artery disease significantly improve psychological outcomes among the patients? A meta-analysis. Medicine (Baltimore). 2018;97(35):e12171. doi: https://doi.org/10.1097/ MD.0000000000012171

\section{PMid:30170464}

30. Corones-Watkins KM, Theobald KA, White KM. Outcomes of a randomised pilot trial of a nurse-led clinic for patients after percutaneous coronary intervention. Aust Crit Care. 2019;32(4):285-92. https://doi.org/10.1016/j.aucc.2018.06.009 PMid:31280772

31. Li C, Liu Y, Xue D, Chan CW. Effects of nurse-led interventions on early detection of cancer: A systematic review and metaanalysis. Int J Nurs Stud. 2020;110:103684. https://doi. org/10.1016/j.ijnurstu.2020.103684

PMid:32702568

32. Akgöz AD, Gözüm S. Effectiveness of a nurse-led physical activity intervention to decrease cardiovascular disease risk in middle-aged adults: A pilot randomized controlled study. J Vasc Nurs. 2020;38(3):140-8. https://doi.org/10.1016/j. jvn.2020.05.002

33. Doherty M, Jenkins W, Richardson H, Sarmanova A, Abhishek A Ashton $\mathrm{D}$, et al. Efficacy and cost-effectiveness of nurse-led care involving education and engagement of patients and a treatto-target urate-lowering strategy versus usual care for gout: A randomised controlled trial. Lancet. 2018;392(10156):140312. https://doi.org/10.1016/S0140-6736(18)32158-5 PMid:30343856

34. Schlottmann H, Broome M, Herbst R, Burkhardt MC, Mescher A. Nurse-led telephone follow-up to improve parent promotion of healthy behaviors in young children with motivational interviewing techniques. J Pediatr Health Care. 2019;33(5):54554. https://doi.org/10.1016/j.pedhc.2019.02.003

PMid:30926151 